FORMATION Formation emploi

Revue française de sciences sociales

128 | octobre-décembre 2014

Pêle-Mêle

\title{
Référentiels et activité : un rapport complexe à améliorer
}

Jean-Paul Cadet

\section{(2) OpenEdition}

Journals

Édition électronique

URL : http://journals.openedition.org/formationemploi/4328

DOI : $10.4000 /$ formationemploi.4328

ISSN : 2107-0946

Éditeur

La Documentation française

Édition imprimée

Date de publication : 31 décembre 2014

Pagination : 101-108

ISSN : 0759-6340

\section{Référence électronique}

Jean-Paul Cadet, «Référentiels et activité : un rapport complexe à améliorer », Formation emploi [En

ligne], 128 | octobre-décembre 2014, mis en ligne le 15 janvier 2015, consulté le 30 octobre 2020.

URL : http://journals.openedition.org/formationemploi/4328 ; DOI : https://doi.org/10.4000/

formationemploi.4328

(c) Tous droits réservés 


\title{
Note de lecture
}

\section{Référentiels et activité : un rapport complexe à améliorer ${ }^{1}$}

\author{
Par Jean-Paul Cadet \\ Chargé d'études en sciences de gestion, Céreq, Département travail, emploi et \\ professionnalisation.
}

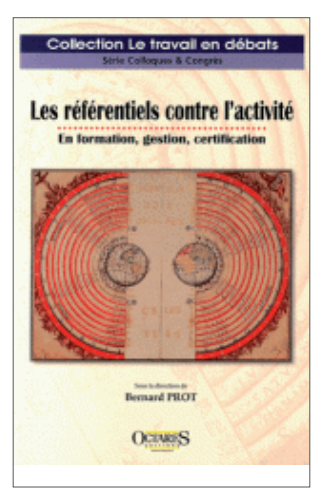

Que ferions-nous sans référentiels ? Aujourd'hui, en France et dans bien d'autres pays comparables, les référentiels sont devenus tellement omniprésents dans les sphères de la formation, de la certification et de la gestion qu'on peine à imaginer un monde et un fonctionnement social qui en seraient totalement dépourvus.

Ces documents visent à formaliser " ce qui fait référence ", c'est-à-dire des repères ou des normes, pour des situations de travail, des emplois ou des métiers donnés. Ils prolifèrent depuis les années 90 , dans un contexte où l'on cherche ou prétend chercher à mieux prendre en compte et reconnaître les activités effectivement exercées, les " compétences » ou les savoirs d'expérience associés à ces activités, au côté des traditionnelles tâches prescrites, connaissances ou savoirs académiques. Les référentiels sont censés aujourd'hui instrumenter et appuyer quantité de processus : la fabrication de diplômes, la conception de programmes de formation, la mise en ouvre des dispositifs de validation des acquis de l'expérience, la gestion de parcours professionnels, mais aussi le management d'activités, la reconnaissance sociale de métiers en émergence ou en forte transformation...

De fait, la forme, le contenu et la méthodologie d'élaboration des référentiels ont tendance à varier sensiblement selon le processus recherché ou l'objectif poursuivi par les concepteurs. Mais les référentiels ont pour point commun de tenter à chaque fois de créer un langage commun et " finalisé » à propos d'une activité de travail. Ils constituent à vrai dire des objets autant sociaux que techniques, élaborés en général par des collectifs d'acteurs et s'adressant de surcroît à des collectifs plus larges d'utilisateurs potentiels. Le rapport

1 Cette note de lecture fait suite à une intervention réalisée lors d'une séance du Séminaire Analyse du Travail-SEMAT du Céreq, le 7 octobre 2014, consacrée à la présentation de l'ouvrage par Bernard Prot. 
entre référentiels et activité reste pourtant problématique. Ce rapport s'avère forcément complexe, car un document réalisé à un moment donné, selon un certain point de vue théorique ou pratique, et dans un cadre d'écriture plus ou moins contraint, ne saurait à l'évidence épouser et représenter toute la richesse et la dynamique d'une activité de travail. Pour autant, il mérite, à n'en pas douter, un examen tout particulier à l'heure de la profusion des référentiels dans les champs de la formation, de la certification et de la gestion des ressources humaines.

D'une part, il convient de s'interroger sur leur degré de convergence ou de divergence avec les activités visées et leurs usages effectifs. D'autre part, on peut se demander dans quelle mesure les analyses scientifiques contemporaines de l'activité pourraient contribuer à favoriser la production de référentiels ajustés au mieux aux métiers ou aux situations " réelles ». C'est précisément ce qu'un groupe de psychologues, de sociologues et de chercheurs en sciences de l'éducation s'est attelé à faire, en publiant l'ouvrage intitulé « Les référentiels contre l'activité ». Celui-ci porte sur le rapport complexe qui existe entre référentiels et activité, et a pour fil directeur ce questionnement à double entrée : "Comment relier le travail de conception de référentiels aux enjeux et méthodes d'analyse de l'activité au travail ? Comment relier des analyses de l'activité réalisées en milieu de travail avec la conception de référentiels?"

Le résultat est dès lors édifiant. Elaboré sous la direction de Bernard Prot, cet ouvrage pluridisciplinaire fait suite à un séminaire qui s'est tenu entre 2010 et 2012. Avec une certaine ouverture sur l'international (une contribution nous vient du Portugal, une autre de Suisse), il aborde le cas de différents types de référentiel et va jusqu'à s'intéresser, pour certains d'entre eux, à leurs usages, alors qu'il est plus courant de traiter la question de leur conception. Il apporte aussi et surtout d'importants éléments, généraux et spécifiques, qui éclairent les relations que les référentiels entretiennent (ou sont susceptibles d'entretenir) avec les activités qu'ils sont censés décrire, normaliser ou appuyer. Un regard transversal et synthétique sur les différentes contributions permet d'identifier ces éléments d'éclairage, tout en invitant à poursuivre la réflexion.

\section{Une critique des référentiels dominants...}

L'ouvrage a d'abord le grand mérite de rappeler et d'approfondir une critique habituellement adressée aux référentiels les plus répandus, à savoir le fait qu'ils se contentent de cartographier et de répertorier des tâches et des compétences. Ils contribuent, de ce fait, à découper les situations de travail, à les séparer entre plusieurs dimensions, alors même que l'activité consiste justement à relier ces dimensions. "L'action réussie n’est pas le produit miraculeux d'une somme d'activités listées dans un référentiel, mais bien le fruit de la mobilisation intégrée, construite, structurée et contextualisée de diverses composantes et activités ", écrit par exemple, à juste titre, Sandrine Cortessis. 
Par ailleurs, beaucoup de référentiels, en particulier les référentiels de compétences en entreprise, ont tendance à se focaliser sur les personnes, leurs capacités cognitives, leurs qualités individuelles, via des énoncés flous et reproductibles dans nombre de situations de travail : « communiquer », « analyser ", « synthétiser »... De fait, ces référentiels s’imposent comme des documents strictement normatifs, destinés avant tout à harmoniser des pratiques et des représentations, à rendre les métiers "indiscutables ", pour reprendre un terme utilisé par Stéphane Balas dans sa contribution.

Cette critique des référentiels dominants est d'autant plus utile qu'ils ne sont pas sans incidence sur les situations et sur ce qui est susceptible d'advenir. Ils peuvent limiter, orienter, contrarier l'action des professionnels amenés à s'en servir. Ils peuvent conduire à des pratiques jugées inappropriées, comme à une «taylorisation des apprentissages » dans le domaine de la formation. Ils peuvent aussi modeler, structurer et figer certaines façons de voir, certaines représentations gestionnaires, comme le suggère Pascal Ughetto à propos des référentiels de compétences élaborés par les gestionnaires des ressources humaines des entreprises, en référence à la théorie des technologies invisibles de gestion ${ }^{2}$.

\section{2 ... mais pas de déterminisme pour autant}

Une telle critique ne débouche pas pour autant sur la mise en évidence systématique d'un déterminisme instrumental. Heureusement, les référentiels ne dictent pas nécessairement l'ensemble des actions et des décisions de leurs utilisateurs, ni a fortiori le devenir des activités qu'ils se sont donnés pour objet, nous dit ainsi en substance l'ouvrage, via quelques contributions.

De fait, malgré leur caractère normatif, ils apparaissent rarement utilisés, et même construits, strictement comme il était prévu qu'ils le soient. Souvent " contournés ", sinon " détournés », ils font alors l'objet d'un important travail de traduction et d'interprétation par les acteurs chargés de les élaborer ou d'en faire usage.

Fabienne Maillard analyse par exemple ce que réalisent les groupes de travail qui conçoivent les « référentiels d'activités professionnelles " (les fameux RAP), destinés à la fabrication des diplômes de l'enseignement professionnel du ministère de l'Education nationale. Elle révèle alors l'intense activité interprétative à laquelle ces groupes s'adonnent pour les élaborer, en regard du guide qui leur est fourni et qui est censé, pour ainsi dire, leur servir de méta-référentiel. Notamment, ils n’hésitent pas à faire surgir et à prendre en compte des

2 Voir à ce sujet Michel Berry, Une technologie invisible? Limpact des instruments de gestion sur l'évolution des systèmes humains, Centre de Recherche en Gestion, Juin 1983. Ce texte fondateur sur la question des outils de gestion n'a jamais été publié. Il peut toutefois être téléchargé sur le site du CRG : http://crg.polytechnique.fr/incunables/techno_invisible.pdf. 
sujets normalement hors champ à ce stade, comme les questions ayant trait à la formation initiale des jeunes.

Autre exemple, analysé par Sandrine Cortessis : le fonctionnement d'un jury chargé de valider des acquis de l'expérience pour des enseignants en Suisse. Dans la construction collective de leur jugement, les jurés prennent leurs distances par rapport au référentiel d'activité qu'ils sont supposés utiliser, et, à force d'exercer ensemble leur fonction de juré, ils en viennent à réinventer une sorte de référentiel implicite guidant leurs prises de décision ${ }^{3}$.

Cette contribution mais aussi dans une certaine mesure celle de Mickaël Huchette sur la recherche de ce que pourrait être un stage idéal dans le champ de l'enseignement technologique et professionnel, montrent qu'il est important que des échanges entre les professionnels s'opèrent et qu'une régulation collective ait lieu autour du projet de référentiel en gestation ou à mobiliser dans l'action. De là dépend en effet en grande partie la possibilité d'un contournement ou d'un détournement du référentiel qui se révèle utile. Ajoutons qu'il semble également souhaitable que les concepteurs des référentiels admettent d'emblée qu'ils soient utilisés avec une marge de réinterprétation et donc une certaine plasticité, comme c'est sans doute le cas, par exemple, au sein de l'Education nationale, avec les référentiels des diplômes de l'enseignement professionnel.

\section{Référentiels à dilemmes et référentiels de situations : deux pistes intéressantes}

Un autre grand atout de l'ouvrage est de relater des expérimentations et d'établir ainsi des propositions pour concevoir autrement les référentiels, afin qu'ils soient plus en correspondance avec les activités.

Sans tomber dans le travers d'une approche qui serait à nouveau normative, ces propositions d'innovation visent à tenir compte de certaines avancées récentes en matière d'analyse de l'activité. Elles démentent, à leur façon, le titre de l'ouvrage, en s'employant à plaider pour des référentiels susceptibles d'être davantage " pour l'activité ». Elles empruntent à deux courants actifs dans le domaine de la psychologie et de l'ergonomie : la didactique professionnelle et la clinique de l'activité.

Adoptant le point de vue de la didactique professionnelle, la première piste évoquée a trait à l'élaboration de référentiels dits "de situations ", à travers les contributions de Régis Ouvrier-Bonnaz, Marianne Lacomblez, Vincent Boccara et al., qui s'appuient

3 Semblable prise de distance par rapport à des référentiels a par exemple été aussi récemment observée à propos du travail d'évaluation des élèves réalisé par les professeurs de l'enseignement professionnel en France : $c f$. Paddeu J. et Veneau P. (2013), « Modes d'évaluation dans les diplômes professionnels ", Céreq, Net.Doc, $n^{\circ} 116$. 
repectivement sur l'exemple du métier de géomètre-expert et le cas d'un référentiel destiné aux formateurs à la conduite automobile. Ces référentiels de situations seraient avant tout compatibles avec ce qui organise ou structure conceptuellement l'activité des opérateurs, les "schèmes " qu'ils mobilisent, et fondés sur une explicitation de l'articulation qui lie situations et "dimensions de compétences" ${ }^{4}$.

Se réclamant de la clinique de l'activité, la seconde piste ouverte, à partir des contributions de Bernard Prot, Stéphane Balas et Pascal Simonet, revient à organiser des dialogues entre des professionnels qui participent collectivement à l'analyse de leur activité, et à repérer à partir de là des "dilemmes génériques " destinés à être pris en considération par les concepteurs de référentiels. Ces dilemmes renvoient à des prescriptions inconciliables qui s'imposent aux professionnels dans leur activité et qui leur demandent de trouver et de mobiliser des ressources individuelles et collectives pour y faire face, au moins provisoirement, par exemple, " administrer des informations et les communiquer à autrui " pour les employés de bureau, procéder aux gestes relatifs aux inhumations en faisant preuve de la meilleure efficience technique possible, tout en prenant en compte le contexte très particulier du rituel funéraire, avec notamment la présence de la famille et des amis du défunt, pour les fossoyeurs. De ce point de vue, les dilemmes représentent de bons matériaux pour concevoir des référentiels aptes à conserver, dans une certaine mesure, la dynamique qui caractérise les activités de travail, à formaliser les tensions et les problèmes irrésolus propres à l'exercice concret de ces activités, et à s'éloigner ainsi des habituelles conceptions qui en font abstraction ou qui les minimisent.

Ces « référentiels à dilemmes » peuvent d'ailleurs être très bien complémentaires des référentiels de situations, si l'on en croit du moins Régis Ouvrier Bonnaz et Marianne Lacomblez, qui proposent de "mettre en scène ", dans les RAP, les " concepts de référence " et les " dilemmes de métier », les seconds étant supposés donner vie aux premiers. Pour les auteurs, ce serait un moyen de mieux cerner les métiers, les connaissances à acquérir et les savoirs à enseigner.

\section{Une invitation à aller plus loin}

L'ouvrage apporte donc beaucoup à l'analyse et à la compréhension du rapport entre référentiels et activité. Mais en même temps, il présente encore, à la lecture, le grand intérêt de ne figer en rien la réflexion et au contraire d'inviter à la poursuivre et à l'étendre, ce qui est une qualité indéniable pour une production à caractère scientifique.

4 Lire aussi à ce sujet Paul Olry, "Le lien travail et formation, un moyen pour apprendre. Selon quel référentiel ? ", in Mahlaoui S. (sous la direction de), Le travail au cour de la relation formation-emploi. Séminaire Analyse du Travail. Journée d'étude, Marseille, 15 octobre 2012, Céreq-Relief, n 45, pp. 31-45. 
Tout d'abord, l'ouverture esquissée en termes de disciplines et de modes d'approche est sans doute loin d'avoir été épuisée pour traiter sous tous ses aspects l'objet de l'ouvrage.

Par exemple, n’y aurait-il pas lieu de faire appel à présent à des spécialistes de la sociologie des sciences et des techniques, des sciences politiques, des sciences de gestion, voire des sciences historiques, ces courants ou ces disciplines ayant concouru d'une manière ou d'une autre à l'analyse des instruments de gestion, parmi lesquels on peut faire figurer les référentiels?

Ensuite, il serait sans doute utile de poursuivre l'exploration des différents types de référentiels existants, ici (dans le vaste océan de la certification, il y a maintenant par exemple vaste matière à réflexion) ou dans d'autres pays, l'ouverture sur l'international amorcée dans l'ouvrage pouvant être perçue comme un début.

L'attention portée aux référentiels élaborés par les entreprises, les administrations ou les branches professionnelles mériterait par ailleurs d'être accentuée. Il n'y a en effet qu'une seule contribution dans l'ouvrage sur ce type de référentiel, celle de Pascal Ughetto, qui porte sur les référentiels de compétences.

De même, il serait certainement opportun de mener des travaux spécifiques sur les formes, les usages et les effets des référentiels qui visent en priorité à appuyer des mobilités et des transitions professionnelles en définissant des correspondances entre différents métiers, alors que ce genre de référentiel se développe un peu partout et de façon hétérogène, au risque d'ailleurs d'entraver pareilles mobilités et transitions ${ }^{5}$.

Une autre façon de prolonger la réflexion pourrait être de continuer à s'intéresser aux " acteurs » des référentiels, dans le sens où leurs logiques d'action, leurs " compétences " et leurs pratiques influent sur la façon dont les référentiels appréhendent et traitent les activités.

On voit bien dans l'ouvrage le rôle prééminent des gestionnaires des ressources humaines dans l'élaboration des référentiels en entreprise, ainsi que certaines facettes du travail d'interprétation et de traduction réalisé par les utilisateurs des référentiels. Il conviendrait maintenant de mettre la focale sur l'activité de leurs concepteurs. Dans la lignée de ce qu'avance l'ouvrage, on devine les difficultés pratiques générées par la conception de tout référentiel. Ce n'est jamais facile de mettre en mots le travail sans trop le circonscrire, mais aussi sans trop le détailler. La difficulté est donc d'ordre technique, mais aussi d'ordre politique, car, comme il a été dit, tout référentiel est un objet sociotechnique ; il est le fruit

5 Pour le cas des répertoires-métiers, $c f$. Cadet J.-P. et Mahlaoui S., "Quand les entreprises et les administrations renouvellent la notion de métier ", Céreq-Bref, n² 282, 2011 ; et d'Agostino A., avec la participation de Séchaud F., Sulzer E. et Vial D., Analyse des outils développés par les Observatoires prospectifs des métiers et des qualifications. Panoramas statistiques et des métiers, Céreq, rapport d'étude pour le COC-CPNFP, consultable et téléchargeable sur le site du Fonds paritaire de sécurisation des parcours professionnels : http:// www.fpspp.org/portail/resource/filecenter/document/042-00001e-02q/1--rapport---panorama-des-basesde-donnees-developpees-par-les-opmq.PDF, 2013. 
des compromis des différents acteurs ayant participé à son élaboration. Les tensions, voire les dilemmes, se font probablement jour dans cette activité de conception, comme par exemple entre le fait de respecter scrupuleusement le cadre technique et méthodologique préalablement conçu et le fait d'accepter les propositions déviantes des différents acteurs sollicités pour édifier le référentiel, afin de favoriser leur implication et leur coordination.

Concernant les propositions établies en matière de référentiels, il apparaît possible, à présent, de s'interroger sur leurs conditions d'extension, sinon de généralisation, dans une perspective d'opérationnalisation ${ }^{6}$.

Plusieurs questions pourraient être ainsi examinées dans cette perspective : comment sont effectivement utilisés les référentiels issus des expérimentations décrites ou alors se réclamant des mêmes principes d'élaboration?

Dans les approches exposées, qui s'appuient sur des démarches de co-analyse impliquant des professionnels des métiers appréhendés, comment s'assurer qu'on ne se trompe pas dans les dimensions de l'activité à explorer et qu'on ne passe pas à côté de situations essentielles qui relèvent de ce qu'on appelle le " travail invisible » ou le "sale boulot », de fait difficilement verbalisables et auto-analysables?

Comment mieux prendre en compte la dimension socio-politique de la conception des référentiels, comme en appelle d'ailleurs de ses vœux Bernard Prot dans sa contribution, après avoir assisté à l'achoppement de deux expériences de référentiels à dilemmes pour des raisons socio-institutionnelles ? Comment procéder concrètement pour passer d'un travail d'analyse et de conception entre "gens de métier " à un travail faisant intervenir les autres acteurs concernés par le métier et son devenir (employeurs, enseignants...) ?

À propos tout particulièrement des diplômes, dans quelle mesure et jusqu'où peut-on expérimenter de nouvelles façons de construire des référentiels alors même que l'ensemble du système est supposé reposer sur une harmonisation ou une cohérence minimales ?

Tandis que les «diplômes-métiers » sont loin aujourd'hui de s'imposer, et ce malgré un certain retour du métier au niveau des CAP, comme le note Fabienne Maillard, comment faire travailler différents « gens de métier » dans la construction d'un même référentiel ?

Le questionnement reste ainsi pleinement ouvert. Sachant qu'il semble possible, de surcroît, d'envisager ou d'examiner d'autres expérimentations qui puisent, cette fois, dans des cadres disciplinaires, théoriques et méthodologiques différents de ceux propres à la

6 Remarquons toutefois que la didactique professionnelle inspire déjà l'élaboration des référentiels des diplômes du ministère de l'agriculture depuis plusieurs années, avec la notion de "situation professionnelle significative ». $C f$. ministère de l'Alimentation, de l'Agriculture et de la Pêche, Guide d'écriture des Référentiels de diplômes professionnels, DRIF-EDUTER-IEA-POFE, 23 décembre 2009. Voir aussi Gilly E., "Discussion ", in Brun G., Crocco M., Mahlaoui S. et Mailliot S. (éds.), Insertion, formation, emploi. Analyse critique des outils conceptuels des professionnels, Céreq-Relief, $\mathrm{n}^{\circ}$ 32, décembre 2010, pp. 52-54. 
didactique professionnelle et à la clinique de l'activitép, on voit donc que, à l'heure où ils continuent à se répandre et se veulent plus que jamais indispensables, la question du rapport des référentiels à l'activité est loin d'être épuisée. Dans l'intérêt partagé des professionnels concernés et des gestionnaires, ce rapport est aujourd'hui à questionner sans cesse pour être amélioré et transformé au mieux. L'ouvrage coordonné par Bernard Prot a sans conteste contribué remarquablement à ouvrir la voie.

Référence de l'ouvrage :

Prot B. (sous la dir.) (2014), Les référentiels contre l'activité, en formation, gestion, certification, Octarès, collection "Le travail en débats », série Colloques et Congrès.

7 A titre d'illustration, il serait sans doute intéressant de réexaminer l'expérimentation d'un référentiel de compétences au sein des secteurs des assurances, dans les années 2000, analysée par une sociologue engagée dans cette opération. Cette expérimentation avait pour originalité de prendre pour objet une activité collective et non plus une activité individuelle, et pour finalité d'élaborer un référentiel destiné explicitement à gérer le travail et non pas l'emploi et les parcours professionnels, à l'instar de la plupart des référentiels de compétences. $C f$. Marie-Christine Combes, «Identifier la dimension collective des compétences pour gérer le travail ", in Jouvenot C. et Parlier M., Elaborer des référentiels de compétences : principes et méthodes, ANACT, 2005, pp. 94-118. 